\title{
Chemische Temperaturregulation bei neugeborenen Mäusen
}

\author{
Josef Pichotka \\ Physiologisches Institut der Universität Bonn
}

\begin{abstract}
Chemical temperature regulation in new-born mice. Immediately after birth, mice are poikilotherm. Changes in environmental temperature cause characteristic variations in metabolic rate. If plotted as a function of rectum temperature, metabolic rate shows the same pattern under conditions of cooling or warming. Below normal rectum temperature, e. g.

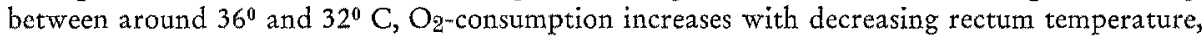
but decreases with increasing rectum temperature. Basically the same relationship between rectum temperature and $\mathrm{O}_{2}$-consumption is found under conditions of constant (for several hours) environmental temperatures. The maximum average $\mathrm{O}_{2}$-consumption is obtained at a constant environmental temperature of $32^{\circ} \mathrm{C}$. Overheating (1 hour at $40^{\circ} \mathrm{C}$ ) destroys the relationship described and results in a simple reduction of $\mathrm{O}_{2}$-consumption with decreasing rectum temperature under conditions of low environmental temperatures. The increasing constancy of body temperature in the second week after birth is caused by the development of the fur on the skin (reduction of heat loss), rather than by that of feed back mechanisms.
\end{abstract}

\section{EINLEITUNG}

Ein Teil der Warmbliiter wird in einem sehr unreifen Zustand geboren. Charakteristische Beispiele dafür sind unter unseren Labortieren Mäuse und Ratten. Eine wirksame Temperaturregulation ist bei diesen Tieren in den ersten Lebenstagen nicht festzustellen. Die Differenz $z$ wischen Körpertemperatur und Umgebungstemperatur ist gering und scheint in weiten Bereichen der Umgebungstemperatur praktisch gleich zu sein. Am Ende der zweiten Lebenswoche entwickelt sich eine zunehmende Unabhängig-" keit der Körpertemperatur von der Umgebungstemperatur. Zur gleichen Zeit kommen die Tiere auch in den Besitz anderer Funktionen, die mit der histologisch nachweisbaren Reifung des Nervensystems zusammenhängen, wie der koordinierten Bewegung, der Fähigkeit zu zittern u. a. Das Erreichen der Homoiothermie bei diesen Tieren wurde daher in gleicher Weise als Folge der Entwicklung des Zentralnervensystems angesehen.

Aus einer Reihe älterer und neuerer Untersuchungen geht hervor, daß auch bei unreif geborenen Tieren bald nach der Geburt Änderungen der Umgebungstemperatur zu charakteristischen Änderungen der Stoffwechselgröße führen. Eine Stoffwechselsteigerung bei unreifen neugeborenen Tieren durch "Abkühlung" ist beim Kaninchen (Giaja 1925, Adamsons 1959), bei Katzen (Leichtentritt 1919, Hil. 1959) und bei 
Hunden (Gelineo 1957, McIntyre \& Ederstrom 1958, Adamsons 1959) beschrieben worden.

Für die Ratte, bei der die Entwicklung zur Homoiothermie in der gleichen Zeit erfolgt wie bei der Maus, hatte ADoLPY (1957) angegeben, daß eine Steigerung der Sauerstoffaufnahme bei Abkühlung erst im Alter von 3 bis 4 Tagen erfolge. Er stützte seine Auffassung dabei im wesentlichen auf die Untersuchungen von ANTOSCHKINA (1939) und FAIRFIELD (1948). In späteren Untersuchungen wurde jedoch nachgewiesen, $\mathrm{da} ß$ auch bei der neugeborenen Ratte in den ersten Lebenstagen eine Steigerung der Sauerstoffaufnahme durch Abkihhlung eintritt (GELINEO \& GELINEO 1951, HAHN \& KoldovsKY 1958, TAYLOR 1960). Die sogenannte chemische Temperaturregulation besteht nach diesen Beobachtungen im Prinzip bereits zu einer Zeit, da die Tiere noch völlig poikilotherm sind.

Um diese in vieler Beziehung erstaunliche Tatsache in unsere Vorstellung von der Temperaturregulation einordnen zu können, ist es zunächst einmal erforderlich, den Umfang und die Bedingungen dieser regulativen Stoffwechselsteigerung festzustellen. Wir haben unsere Untersuchungen an Mäusen durchgeführt. Ein Teil der Ergebnisse liegt in vorläufigen Veröffentlichüngen vor (РіснотKA 1960, 1961, CHOINOwSKI 1962, HOHNSTÄDTER 1962). Von CASSIN (1963) wurde inzwischen bei neugeborenen Mäusen ebenfalls das Auftreten einer Stoffwechselsteigerung bei Abkühlung beschrieben.

\section{METHODIK}

Die Versuche wurden an weißen Mäusen (Stamm Agnes Bluhm) von wenigen Stunden nach der Geburt bis zum Alter von 5 Tagen durchgeführt. Die Elterntiere befanden sich mit den Neugeborenen in einem auf $22^{\circ} \pm 1,0^{\circ} \mathrm{C}$ geregelten Raum. Das
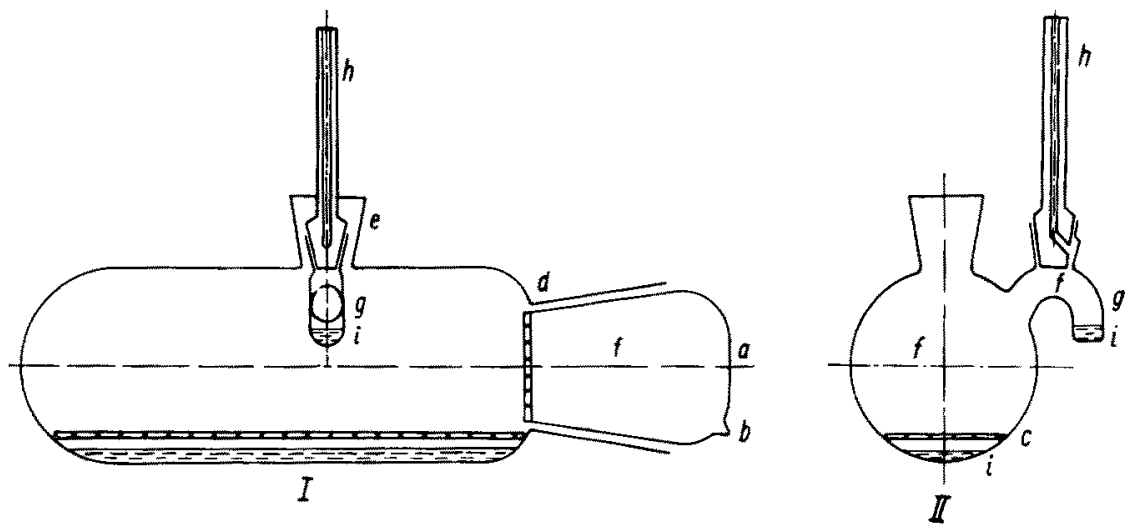

Abb. 1: Reaktionsgefäß zur Stoffwedıselmessung junger Mäuse

Körpergewicht der jungen Tiere lag je nach dem Alter zwischen 1,2 und 3,2 g. Die $\mathrm{O}_{2}$-Aufnahme wurde manometrisch in der Warburg-Apparatur gemessen. Dazu wurden spezielle Reaktionsgefäße von liegender Zylinderform mit $120 \mathrm{~cm}^{3}$ Inhalt benutzt (Abb. 1). Die zur Absorption der $\mathrm{CO}_{2}$ erforderliche Kalilauge befand sich unter einem 
perforierten Boden, der über die ganze Länge in das Gefäß eingezogen war. Als Thermobarometer dienten Reaktionsgefäße gleicher Abmessungen; an Stelle der KOH enthielten sie die gleiche Menge Wasser. Die Messungen erfolgten in atmosphärischer Luft. Der verbrauchte Sauerstoff wurde bei der bestehenden Temperatur mit einer besonderen Einrichtung je nach der Verbrauchsgröße alle 10 bis 20 Minuten dem Reaktionsgefäß wieder zugeführt. Auf diese Weise war praktisch keine Zeit für den Temperaturausgleich notwendig. Die $\mathrm{O}_{2}$-Spannung im Reaktionsgefäß bewegte sich zwischen 19 und $21 \%$.

In den bisher vorliegenden Untersuchungen wurde im allgemeinen so vorgegangen, daß zunächst die sogenannte thermoneutrale Zone für die betreffende Spezies festgestellt wurde, d. h. die Umgebungstemperatur, bei der der niedrigste Stoffwechsel beobachtet wurde. Im Vergleich dazu wurde der Stoffwechsel bei einer konstanten niedrigeren Umgebungstemperatur gemessen; diese zweite Umgebungstemperatur wurde so gewählt, daß eine möglichst hohe $\mathrm{O}_{2}$-Aufnahme resultierte (AntoschKinA. 1939, Gelineo \& Gelineo 1951, Barić 1953, Taylor 1960).

Auf Grund der Erfahrung in früheren Untersuchungen an erwachsenen Tieren (PichotKa 1953, 1961) gehen die folgenden Versuche von der Möglichkeit aus, daß die Stoffwechselgröße der Neugeborenen eine charakteristische Funktion der Körpertemperatur ist. Daher wurden nicht zwei feste Umgebungstemperaturen als Meßsituation gewählt, sondern die Umgebungstemperaturen wurden in einem weiten Bereich stetig verändert. Die dadurch bedingte Anderung der Körpertemperatur und der $\mathrm{O}_{2}$-Aufnahme wurde fortlaufend gemessen. Bei der Wahl der Anderungsgeschwindigkeit der Temperatur ist zu berücksichtigen, daß offensichtlich eine wesentliche Zeit erforderlich ist, bis die der jeweiligen Umgebungs- und Körpertemperatur zugehörige Stoffwechselgröße erreicht ist. Aus diesem Grund erschien es günstig, die Temperaturänderungen möglichst langsam durchzuführen. Andererseits behalten die jungen Tiere nur für eine bestimmte Zeit nach der Fütterung ihre volle Leistungsfähigkeit. Unter Berücksichtigung dieser Bedingungen wurde eine stetige Anderung der Umgebungs"temperatur von $0,06^{0}$ pro Minute gewählt. Sie wurde durch eine kontinuierliche Verstellung der Kontaktthermometer an den Thermostaten erreicht. Aus Gründen, die in. der Untersuchung klargelegt werden, wurden die Messungen im allgemeinen bei $\mathrm{Ab}$ kühlung der Thermostatentemperatur von $38^{\circ}$ nach $24^{\circ} \mathrm{C}$ oder bei Aufwärmung über den gleichen Bereich durchgeführt. Je nach der Dauer der Vor- und Nachperiode lagen die Versuchszeiten $z$ wischen 5 und 8 Stunden. Die $\mathrm{O}_{2}$-Aufnahme wurde alle 5 Minuten abgelesen; die Thermobarometerwerte bildeten die Nullwerte für die Ablesung.

Die Messung der Körpertemperatur erfolgte rektal mit einem feinen Thermoelement aus Kupfer und Konstantan, das $1 \mathrm{~cm}$ tief eingeführt und durch feine Heftpflasterstreifen fixiert wurde. Das Vergleichselement befand sich in einem Thermostaten von $30^{\circ} \mathrm{C}$.

Die Jungen wurden unmittelbar vor der Messung aus dem Nest entnommen und nach Beendigung des Versuches wieder in das Nest zurüdkgegeben. Sie wurden regelmäßig von der Mutter wieder angenommen, so daß auch wiederholte Messungen an demselben Tier keine Schwierigkeiten bereiteten. Zum Vergleich wurden immer Tiere desselben Wurfes herangezogen. Die Wurfgrößen lagen im allgemeinen zwischen 6 und 10 Tieren; in den meisten Fällen waren es 8 Tiere. 


\section{ERGEBNISSE}

Bei kontinuierlicher Abkïhlung und Aufwärmung der Tiere bewegt sich die Rektaltemperatur (RT) praktisch mit der gleichen Steilheit wie die Umgebungstemperatur (UT). Im Bereich der UT von $24^{\circ}$ bis $38^{\circ} \mathrm{C}$ lag die beobachtete RT $1^{\circ}$ bis $2^{\circ} \mathrm{C}$ über der UT. Im landläufigen Sinne waren diese Tiere mithin vollkommen poikilotherm.

Anderungen der Stoffwechselgröße fanden sich sowohl bei der kontinuierlichen Erhöhung der UT von $24^{\circ}$ nach $38^{\circ} \mathrm{C}$ wie bei der Abkühlung von $38^{\circ}$ nach $24^{\circ} \mathrm{C}$. Diese Anderungen der Stoffwechselgröße sind in den folgenden Diagrammen als Funktion der RT angegeben. Die Berechtigung zu diesem Verhalten ergibt sich aus der Dar-

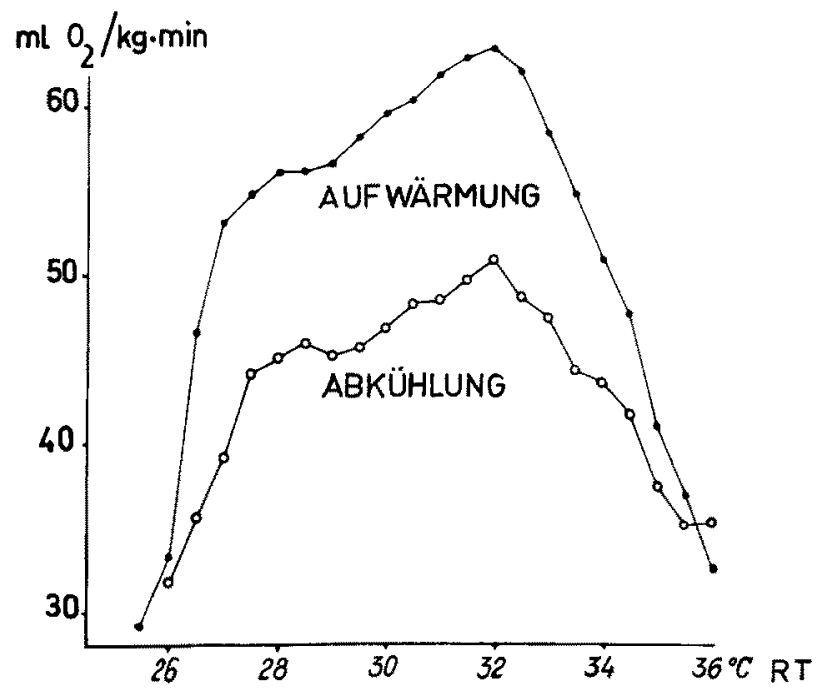

Abb. 2: Die $\mathrm{O}_{2}$-Aufnahme als Funktion der Rektaltemperatur (RT) bei neun bzw. acht 5 tägigen Tieren bei Aufwärmung und Abkühlung. Für beide Versuchsbedingungen ergibt sich das gleiche Bild. Bei der Ablsühlung ist dabei mit Regelmäßigkeit die Og-Aufnahme etwas geringer als bei der Aufwärmung

stellung der Abbildung 2. Es handelt sich um die Mittelwerte der Stoffwechselgröße bei Abkühlung und Aufwärmung 5tägiger Tiere über den gleichen Temperaturbereich. Der allgemeine Verlauf der Stoffwechselgröße über der RT ist für beide Gruppen völlig übereinstimmend. Die erreichte absolute Stoffwechselgröße ist bei den Tieren, die von niedrigeren nach höheren Temperaturen erwärmt werden, in allen Versuchsreihen größer als für die Tiere, die über den gleichen Temperaturbereich abgekühlt werden.

Aus späteren Messungen ist es wahrscheinlich geworden, daß die niedrigere Stoffwechselgröße der zweiten Gruppe darauf zurückzuführen ist, daß die Tiere vor Beginn der Messungen sich für etwa 1 Stunde in der hohen UT befanden. Unabhängig davon, ob es sich um Messungen bei Abkühlung oder Aufwärmung handelt, liegt ein ausgeprägtes Maximum der $\mathrm{O}_{2}$-Aufnahme um $32^{\circ} \mathrm{C}$ und ein relatives Maximum bei $28^{\circ} \mathrm{C}$. Die Stoffwechselgröße liegt im Maximum zum Teil mehr als doppelt so hoch als bei den hier zum Vergleich herangezogenen höheren (ca. $36^{\circ} \mathrm{C}$ ) und niedrigeren (ca. $26^{\circ}$ C) Rektaltemperaturen. 
Wegen der günstigen Bedingungen ist im allgemeinen die Messung der R'T und der O-Aufnahme bei Erhöhung der UT von $24^{\circ}$ nach $36^{\circ}$ oder $38^{\circ} \mathrm{C}$ gewählt worden. Das Ergebnis solcher Messungen an einer Gruppe von eintägigen Tieren ist als Streudiagramm in der Abbildung 3 enthalten. Aus dieser Abbildung ergibt sich, daß in allen Fällen ein Anstieg der Stoffwechselgröße mit steigender Temperatur erfolgt bis zu

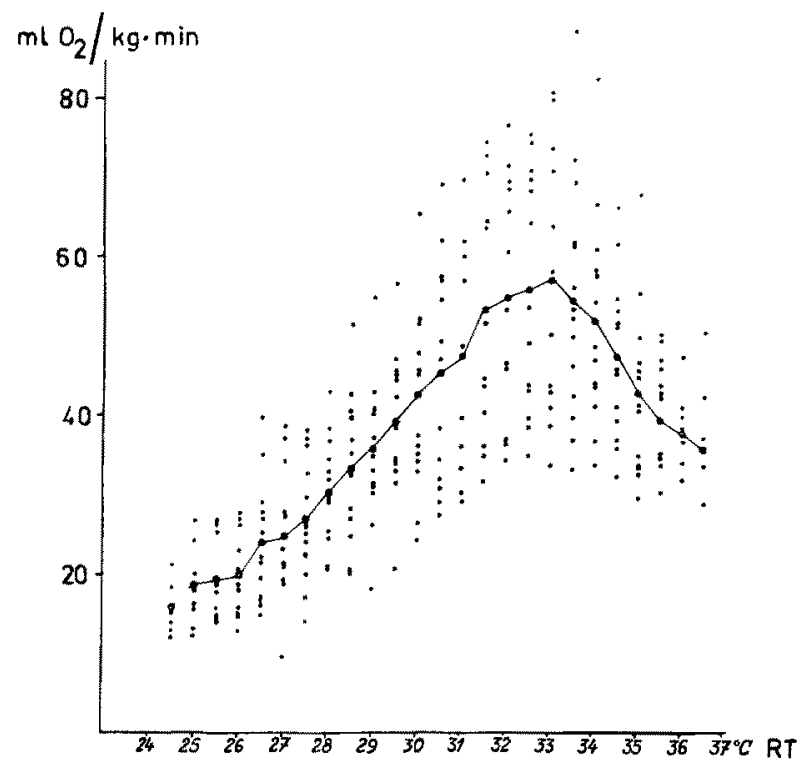

Abb. 3: Streudiagramm der $\mathrm{O}_{2}$-Aufnahme von neun 1tägigen Tieren bei kontinuierlicher Anderung der Umgebungstemperatur (UT) von $24^{\circ}$ auf $36^{\circ} \mathrm{C}$. Die O2-Aufnahme ist dargestellt als Funktion der RT. Aus der Darstellung ergibt sich, daß ein deutliches Maximum der $\mathrm{O}_{2}$ Aufnahme im Bereich der RT von etwa $32^{\circ}$ bis $34^{\circ} \mathrm{C} z u$ beobachten ist

einem Maximum zwischen $32^{\circ}$ und $34^{\circ} \mathrm{C}$ rektal. Danach fällt die $\mathrm{O}_{2}$-Aufnahme mit weiter steigender Temperatur charakteristisch $a b$. Dieser Verlauf drückt sich in der Mittelwertskurve klar aus.

Mit dem Alter der Tiere ändert sich im wesentlichen nur die absolute Höhe des Stoffwechsels; die charakteristische Beziehung von $\mathrm{O}_{2}$-Aufnahme und $\mathrm{RT}$ bleibt unverändert. In der Abbildung 4 finden sich die Mittelwerte für eine Gruppe von 9 Tieren, für die vollständige Messungen am 1., 3. und 5. Lebenstag vorliegen. In allen drei Kurven findet sich der grundsätzlich gleiche Verlauf mit einem deutlichen Maximum in der Nähe von $32^{\circ} \mathrm{C}$ rektal. Die Übereinstimmung wird noch deutlicher, wenn man für die Darstellung die Stoffwechselgröße pro Einheit Körpergewicht benutzt. Das ist in der folgenden Abbildung 5 für die Messungen am 1. und 5. Lebenstag geschehen. In beiden Kurven findet sich der steile Anstieg und Abfall der Stoffwechselgröße im selben Temperaturbereich. Der Abfall der $\mathrm{O}_{2}$-Aufnahme mit steigender Temperatur nach Uberschreiten des Maximums deckt sich in beiden Gruppen weitgehend. Daneben ist zu bemerken, daß insgesamt die Stoffwechselsteigerung bei den 1tägigen Tieren geringer ist als bei den 5 tägigen. 
Aus den berichteten Messungen bei kontinuierlicher Abkühlung oder Aufwärmung ergibt sich mithin eine charakteristische Bezichung zwischen Körpertemperatur und Stoffwechselgröße. Für das hier in Frage stehende Problem interessiert insbesondere das Verhalten in einem bestimmten Abschnitt. Von etwa $36^{\circ} \mathrm{C}$ abwärts, also in dem Temperaturbereich, der unmittelbar unter der normalen Körpertemperatur der Spezies

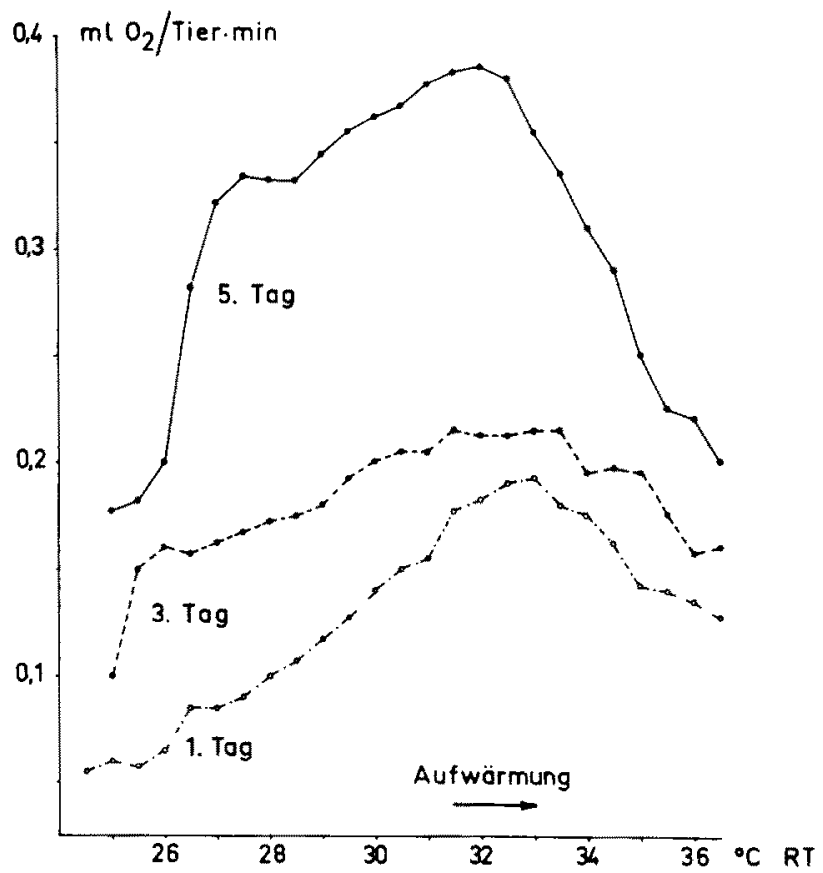

Abb. 4: Die OZ-Aufnahme als Funktion der RT bei neugeborenen Mäusen. Mittelwertskurve von 9 Tieren, deren $\mathrm{O}_{2}$-Aufnahme am 1., 3. und 5. Lebenstag während der Aufwärmung gemessen wurde. Abszisse: beobachtete Rektaltemperatur; Ordinate: $\mathrm{O}_{2}$-Aufnahme. Die $\mathrm{O}_{2}$-Aufnahme stellt sich in allen drei Versuchsgruppen als eine charakteristische Funktion der RT dar, mit einem Maximum bei $32^{\circ} \mathrm{C}$

liegt, steigt die Stoffwechselgröße mit fallender Temperatur steil an. Sie erreicht im allgemeinen bei Temperaturen $z$ wischen $34^{\circ}$ und $30^{\circ} \mathrm{C}$ ein mehr oder minder ausgedehntes Maximum und fällt dann mit weiter fallender Temperatur $a b$.

Diese bei gleitender Temperaturänderung gewonnene Beziehung zwischen Körpertemperatur und Stoffwechselgröße läßt sich auch bei konstanten Umgebungs- bzw. Körpertemperaturen nachweisen. Zu diesem Zweck wurden Gruppen von jeweils 10 Tieren für 4 Stunden nach konstanten Umgebungstemperaturen zwischen $24^{\circ}$ und $36^{\circ} \mathrm{C}$ versetzt. Nach einer mehr oder minder langen Einstellzeit erreichten diese Tiere bei den gegebenen Umgebungstemperaturen konstante Körpertemperaturen und konstante $\mathrm{O}_{2}$-Aufnahmen. Die Einstellzeiten bis zum Erreichen der konstanten $\mathrm{O}_{2}$-Aufnahme und der konstanten Körpertemperatur waren um so größer, je höher die $\mathrm{O}_{2-}$ Aufnahme bei der betreffenden Bedingung war und je größer die Differenz Rektaltemperatur : Umgebungstemperatur wurde. Generell läßt sich feststellen, daß für alle 
Versuchsgruppen die Differenz Rektaltemperatur : Umgebungstemperatur direkt proportional der $\mathrm{O}_{2}$-Aufnahme ist. Die Einstellyerläufe von Rektaltemperatur und $\mathrm{O}_{2}$ Aufnahme bei den zumeist interessierenden Umgebungstemperaturen von $32^{\circ}, 34^{\circ}$ und $36^{\circ} \mathrm{C}$ sind in den Abbildungen 6 und 7 dargestellt. Die Utbereinstimmung sowohl des Einstellverlaufes als auch der erreichten Endwerte beider Größen ist offensichtlich. In der Abbildung 8 sind alle im Gleichgewicht erreichten Werte für die $\mathrm{O}_{2}$-Aufnahme und die Differenz RT - UT eingetragen. Aus dieser Darstellung ergibt sich zunächst einmal

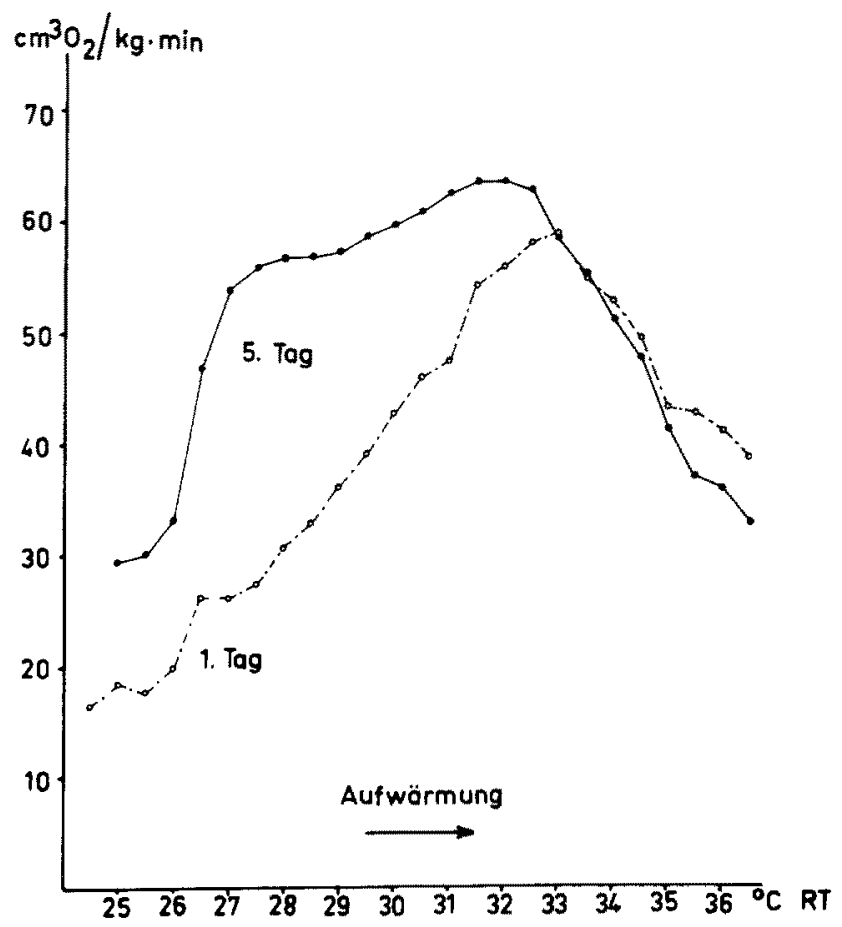

Abb. 5: Mittelwertskurve der $\mathrm{O}_{2}$-Aufnahme pro $\mathrm{kg} \cdot \min$ für 9 Tiere am 1. und 5. Lebenstag. Die gleichen Messungen wie in der vorhergehenden Abbildung. Insgesamt ist die Steigerung der $\mathrm{O}_{2}$-Aufnahme bei den 5 tägigen Tieren höher als bei den 1tägigen. Die steilen Änderungen der $\mathrm{O}_{2}$-Aufnahme erfolgen in beiden Kurven in den gleichen engen Temperaturintervallen

überzeugend die direkte Proportionalität des Gradienten RT - UT zur $\mathrm{O}_{2}$-Aufnahme. Diese Proportionalität bedeutet, daß keinerlei Änderung der Wärmeabgabe bei diesen Beobachtungen im Spiel sein kann.

Darüber hinaus demonstriert die Abbildung 8 die praktisch völlige Übereinstimmung mit der bei gleitender Temperatur gemessenen Beziehung von Körpertemperatur und $\mathrm{O}_{2}$-Aufnahme. Um die in der Abbildung 8 dargestellten Ergebnisse mit den Abbildungen 2, 4 und 5 vergleichbar $z \mathfrak{u}$ machen, muß zu dem Wert für die Umgebungstemperatur auf der Abszisse jeweils die gleichzeitig bestehende Differenz RT : UT addiert werden. Das Maximum der $\mathrm{O}_{2}$-Aufnahme bei konstanten Umgebungsbedingungen und dadurch konstanten Körpertemperaturen der neugeborenen Mäuse liegt somit ebenfalls im Bereich von $32^{\circ}$ bis $34^{\circ} \mathrm{C}$ rektal. Aus den mehrstiindigen Messungen 


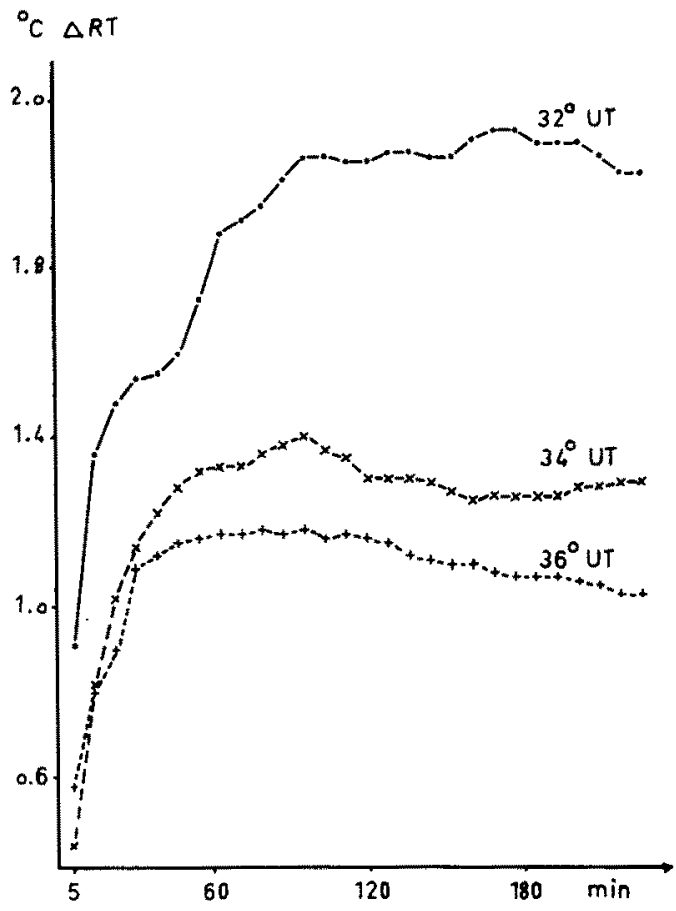

Abb. 6: Mittelwertskurven der RT von 3tägigen Mäusen (jeweils 10 Tiere) nach dem Versetzen in eine konstante Umgebungstemperatur von $32^{\circ}, 34^{\circ}$ und $36^{\circ} \mathrm{C}$. Die RT ist angegeben als Differenz zur konstanten Thermostaten-Temperatur. Aus dem Diagramm ergibt sich, daß die RT nach einer bei den einzelnen Umgebungsbedingungen verschiedenen Einstellzeit auf dem erreichten Niveau konstant bleibt. Die höchste Differenz, nämlich von $2^{\circ} \mathrm{C}$, findet sich bei einer $\mathrm{UT}$ von $32^{\circ} \mathrm{C}$; bei $34^{\circ}$ und $36^{\circ} \mathrm{C}$ liegen die Werte kontinuierlich tiefer

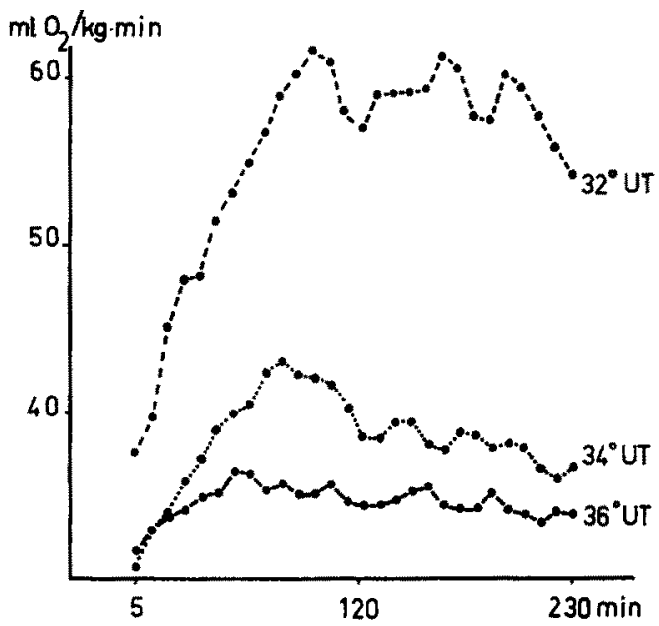

Abb. 7: Mittelwertskurven der $\mathrm{O}_{2}$-Aufnahme von 3 tägigen Mäusen (jeweils 10 Tiere) nach dem Versetzen in konstante Umgebungstemperaturen. Der Verlauf der $\mathrm{O}_{2}$-Aufnahme zeigt dasselbe Bild wie die RT im vorhergehenden Diagramm. Die höchste $\mathrm{O}_{2}$-Aufnahme findet sich bei $32^{\circ} \mathrm{C}$; bei $34^{\circ}$ und $36^{\circ} \mathrm{C}$ liegen die Werte zunehmend tiefer. Die $\mathrm{O}_{2}$-Aufnahme bleibt bei jeder der angegebenen Bedingungen für mehrere Stunden konstant 
bei konstanten Umgebungsbedingungen ergibt sich mithin die gleiche Beziehung zwischen Rektaltemperatur und $\mathrm{O}_{2}$-Aufnahme, wie wir sie bei kontinuierlich gleitender Abkühlung und Aufwärmung gemessen haben.

Die Regelung der Körpertemperatur über die Wärmebildung setzt eine prinzipielle Charakteristik der Art voraus, wie sie in den Abbildungen 2, 3, 4, 5 und 8 zum Vorschein kommt, nämlich daß innerhalb eines bestimmten Temperaturintervalls die Wärmebildung mit fallender Temperatur steigt. Durch eine Reihe zufälliger Erfahrungen gelang es nachzuweisen, daß diese regulative Stoffwechselsteigerung bei in bestimmter Weise geschädigten 'Tieren vollständig ausfallen kann. Es wurde schon betont, $\mathrm{da}$ die Stoffwechselsteigerung bei der Abkühlung zuvor aufgewärmter Tiere immer zu

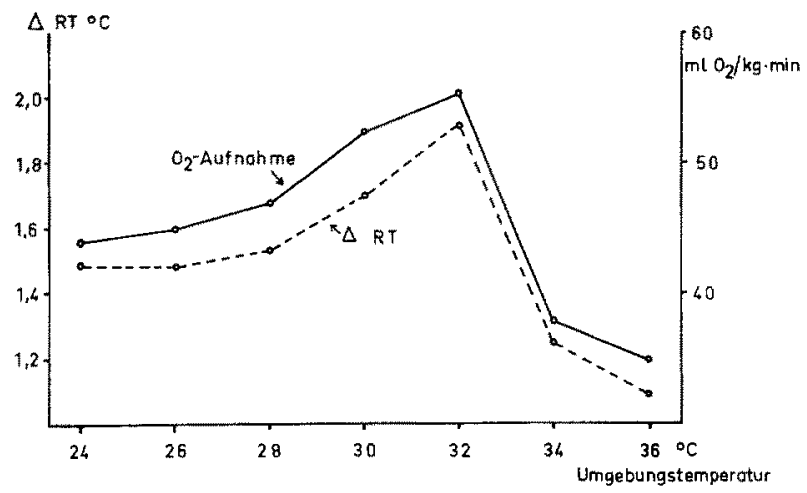

Abb. 8: Zusammenfassung von Messungen bei konstanten UT von $24^{\circ}$ bis $36^{\circ} \mathrm{C}$, wie sie im zeitlichen Verlauf in den Abbildungen 6 und 7 dargestellt sind. Mittelwerte von jeweils 8 bis 10 Tieren. Angegeben ist die $\mathrm{O}_{2}$-Aufnahme und die RT als Differenz zur Ungebungstemperatur, wie sie im eingestellten Zustand erreicht wurden. Die höchste $\mathrm{O}_{2}$-Aufnahme findet sich bei einer UT von $30^{\circ}$ bis $32^{\circ} \mathrm{C}$, d. h. einer RT von $32^{\circ}$ bis $34^{\circ} \mathrm{C}$. Die Differenz RT : UT geht immer mit der gleichzeitigen $\mathrm{O}_{2}$-Aufnahme streng parallel

niedrigeren Werten führte als bei der Aufwärmung der Tiere von niedrigen Tempeturen aus. Wenn die Abkühlung bei Umgebungstemperaturen von $39^{\circ}$ oder $40^{\circ} \mathrm{C}$ begann, starb ein Teil der Tiere während der Experimente oder kurz danach. Nur die robusteren und darunter insbesondere die 1- bis 2 tägigen Tiere überlebten. Bei diesen überwärmten Tieren fand sich eine ganz andere Beziehung zwischen Rektaltemperatur und Stoffwechselgröße. Die Og-Aufnahme fiel bei Abkühlung praktisch als stetige Funktion der Rektaltemperatur. Der in allen vorherigen Messungen beschriebene Anstieg der Stoffwechselgröße von $36^{\circ} \mathrm{C}$ abwärts fehlte bei diesen Tieren vollständig. Diese Beobachtungen sind in Abbildung 9 enthalten. Es handelt sich bei der Kurve der geschädigten Tiere um den Mittelwert von 9 drei- bis fünftägigen Tieren, die nach voraufgehender Überwärmung ( 1 Stunde bei $40^{\circ} \mathrm{C}$ ) kontinuierlich abgekühlt wurden. Die $\mathrm{O}_{2}$-Aufnahme fällt ohne eine erkennbare regulative Bewegung stetig mit der $\mathrm{RT}$ ab. Die Kurve der nicht geschädigten Tiere ist der Mittelwert einer gleichzeitig gemessenen Gruppe von 3- bis 5tägigen Mäusen bei der Aufwärmung von $26^{\circ}$ nach $38^{\circ} \mathrm{C}$ mit der charakteristischen gegenläufigen Bewegung von $\mathrm{O}_{2}$-Aufnahme und RT im Bereich unterhalb $36^{\circ} \mathrm{C}$.

Das Ergebnis dieser Messungen ist also die Feststellung, daß schon bei der neu- 
geborenen Maus offensichtlich in den ersten Stunden nach der Geburt die Stoffwechselgröße innerhalb eines bestimmten Bereichs mit fallender Temperatur steigt. Daß diese Steigerung der Stoffwechselgröße zu keiner Stabilisierung der Körpertemperatur führt, liegt offensichtlich an dem Fehlen eines hinreichenden Wärmewiderstandes und an dem Fehlen jeglicher physikalischen Regulation. Die Tatsache, daß bei neugeborenen Mäusen in der zweiten Woche eine zunehmende Stabilität der Körpertemperatur und ein allmählicher Übergang zur Homoiothermie erfolgt, liegt nicht daran, daß sich in dieser Zeit besondere Regulationsmechanismen ausbilden, sondern hat seine Ursache

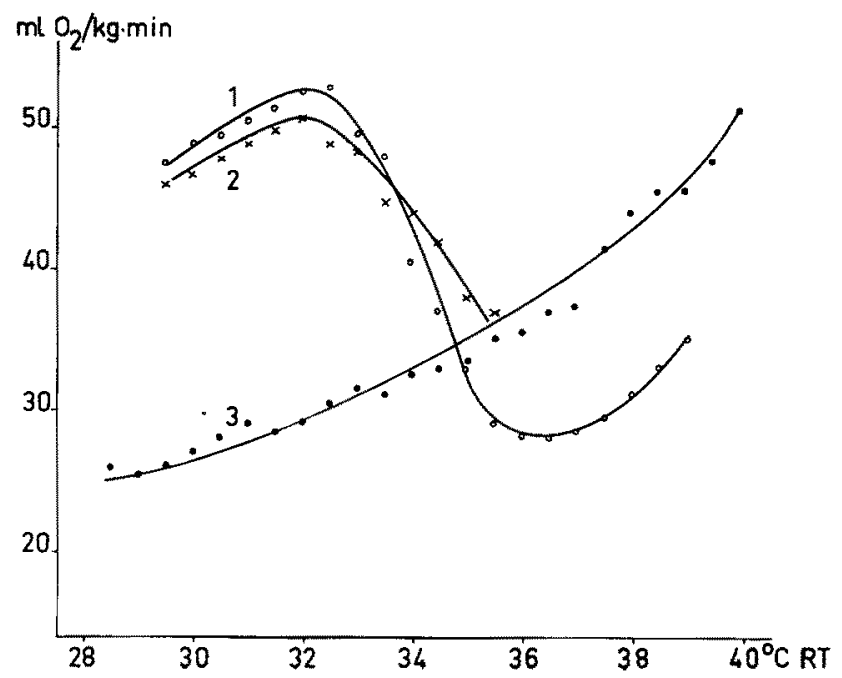

Abb. 9: Die O2-Aufnahme bei neugeborenen Mäusen als Funktion der RT-Mittelwertskurven. 1: Gesunde Tiere bei Aufwärmung von $28^{\circ}$ nach $38^{\circ} \mathrm{C}$ (5 Tiere); 2: Gesunde Tiere bei Abkuhlung von $36^{\circ}$ nach $26^{\circ} \mathrm{C}$ (8 Tiere); 3: Abkühlung überwärmter Tiere von $40^{\circ}$ nach $28^{\circ} \mathrm{C}$. Alle Tiere drei- bis fünftägig

vielmehr darin, daß mit dem sich jetzt entwickelnden Fell ein hinreichender Wärmewiderstand entsteht, an dem die regulative Steigerung der Wärmebildung wirksam wird. Es ist anzunehmen, daß in der gleichen Zeit physikalische Regulationsmechanismen hinzukommen, wenngleich bisher keine positiven Beobachtungen dazu bekannt geworden sind.

\section{ZUSAMMENFASSUNG}

1. Neugeborene Mäuse sind in den ersten Lebenstagen praktisch völlig poikilotherm.

2. Bei kontinuierlicher Abkühlung oder Erwärmung finden sich charakteristische Stoffwechseländerungen. Wenn die beobachtete Stoffwechselgröße als Funktion der Rektaltemperatur (RT) dargestellt wird, ergibt sich für Abkühlung und Erwärmung dasselbe Bild. Wesentlich ist, daß im Bereich unterhalb der normalen RT (also von etwa $36^{\circ}$ bis $32^{\circ} \mathrm{C}$ ) die $\mathrm{O}_{2}$-Aufnahme mit fallender $\mathrm{RT}$ ansteigt, beziehungsweise mit steigender Temperatur abfällt.

3. Dieselbe grundsätzliche Beziehung zwischen $\mathrm{RT}$ und $\mathrm{O}_{\mathrm{g}}$-Aufnahme ergibt sich aus Versuchen bei für mehrere Stunden konstanten Umgebungstemperaturen. Bei einer 
Umgebungstemperatur (UT) von $32^{\circ} \mathrm{C}$ findet sich im Mittel die höchste $\mathrm{O}_{2}$-Aufnahme; oberhalb und unterhalb ist die $\mathrm{O}_{2}$-Aufnahme geringer.

4. Die charakteristische Beziehung zwischen $\mathrm{RT}$ und $\mathrm{O}_{2}$-Aufnahme, die beim gesunden jungen Tier vorliegt, läßt sich durch Überwärmen beseitigen. Nach einstündigem Aufenthalt bei $40^{\circ} \mathrm{C}$ fand sich bei Abkühlung ein einfacher Abfall der $\mathrm{O}_{2}$-Aufnahme mit der RT.

5. Die zunehmende Konstanz der Körpertemperatur in der zweiten Lebenswoche kommt nicht durch die dann einsetzende Entwicklung von Regelmechanismen zustande, sondern durch die Entstehung eines Wärmewiderstandes in Gestalt des Felles.

\section{ZITIERTE LITERATUR}

Adamsons, K., 1959. Breathing and the thermal environment in young rabbits. J. Physiol. 149, $144-153$.

AdoLPH, E. F., 1957. Ontogeny of physiological regulations in the rat. Quart. Rev. Biol. 32, 89-137.

Antoschkina, E. D., 1939. Uber die Ausbildung der Wärmeregulierung im Laufe der Ontogenese. Secherrow Physiol. J. USSR. 26, 1-15.

Barić, I., 1953. La consummation d'oxygène du rat nouveau-né au cours du jeune. Bull. Acad. serbe Sci. Cl. Sci. math. nat. 12, 71-76.

Cassin, S., 1963. Critical oxygen tensions in newborn, young, and adult mice. Am. J. Physiol. $205,325-330$.

CHonowski, H., 1962. Beziehungen zwischen Sauerstoffaufnahme und Körpertemperatur bei neugeborenen weißen Mäusen. Math. nat. Diss. Greifswald.

FAIRFIELD, J., 1948. Effects of cold on infant rats: body temperatures, oxygen consumption, electrocardiograms. Am. J. Pbysiol. 155, 355-365.

Gexineo, S., 1957. Développement ontogénétique de la thermorégulation chez le chien. Bull. Acad. serbe. Sci. Cl. Sci. math. nat. 18, 97-122.

- \& Gelineo, A., 1951. Sur la thermorégulation du rat nouveau-né et la température du nid. C. भ. Acad. bebd. Séanc. Acad. Sci., Paris, 232, 1031-1032.

Graja, J., 1925. Le métabolisme du sommer et le quotient métabolique. Annls Pbysiol. Physicochim. biol. 1, 596-627.

HaHN, P. \& Koldovsкy, O., 1958. Singnificance of the adrenal glands during the post-natal development of thermoregulation in the rat. Nature, Lond. 181, 847.

Hrix, J. R., 1959. The oxygen consumption of new-born and adult mammals. Its dependence on the oxygen tension in the inspired air and on the environmental temperature. $J$. Physiol. $149,346-373$.

HohnstäDTER, G., 1962. Untersuchung über die Beziehung von Stoffwechsel und Muskelaktionsströmen in Abhängigkeit von der Rektaltemperatur. Diss. Physiol. Inst. der Humboldt-Univ. Berlin.

Leichtentritt, B., 1919. Die Wärmeregulation neugeborener Säugetiere und Vögel. Z. Biol. $69,545-563$.

McIntyre, D. G. \& Ederstrom, H. E., 1958. Metabolic factors in the development of homeothermy in dogs. Am. J. Physiol. 194, 293-296.

PrсноткA, J., 1953. Die Bedeutung der Schilddrüse für die Temperaturregulation. Arch. exp. Path. Pharmak. 220, 398-413.

- 1960. Die Beziehung zwischen Körpertemperatur und Stoffwechselgröße bei neugeborenen Nagern. Pflügers Arch. ges. Physiol. 272, 26.

- 1961. Die Temperaturabhängigkeit des Stoffwechsels von Gewebe neugeborener Nager. Pflügers Arch. ges, Physiol. 274, 72.

TAYLOR, P. M., 1960. Oxygen consumption in new-born rats. J. Physiol. 154, 153-168. 